\title{
Do Animals Make Art or the Evolutionary Continuity of Species: A Case for Uniqueness
}

\author{
Jerzy Luty \\ University of Environmental and Life Sciences, Wroclaw \\ jerzyluty@gmail.com
}

Received 9 August 2016; accepted 2017.

\begin{abstract}
When Władysław Tatarkiewicz wrote that there are only two things that can be said about art: that it is a human activity, not a product of nature, and that it is a conscious activity (or its product), adding that every statement about art different from the ones mentioned above was always finally overthrown (Tatarkiewicz, 1980, p. 37), he probably did not think that the first claim could be questioned by anyone. In the following paper, I will trace the history of observations of "artistic behaviors" that were made by animal ethologists and then processed by evolutionary art philosophers who may lead to the hypothesis about the validity of assigning artistic abilities to animals. In respect to this article is aimed at a wide audience. I will also demonstrate that the question: whether, and in what sense, animals create art is in fact a question about a definition of art that could include this type of intentional animal acts.
\end{abstract}

Keywords: evolutionary aesthetics; animal-made art; ethology; art definition.

The contemporary discussion about the possibility of the existence of animal-made art (animal art debate) originates from the letter of Julian Huxley to Nature, in which the British naturalist describes a London zoo gorilla tracing the outline of its own shadow. Because, as Huxley notes, the gorilla did it at least three times, he recognizes in it "possible sources of human graphic art" (de Wall, 2001; Morris, 2013). Nadia Ladygina Kohts who in the 1920s investigated the perception of color and shape in young chimpanzees, observed the enthusiasm with which they draw with pencil on paper. In turn, Paul Schiller in 
the 1940s conducted simple experiments where chimpanzees completed ready-made patterns on a piece of paper and did so in a way that indicated a sense of symmetry and ability to control the composition. The real breakthrough, however, was the experiment that a student of Nikolaas Tinbergen, Desmond Morris, conducted at the Zoological Society of London in the years 1956-1958 with the participation of a chimp named Congo.

According to Morris, the author of such books as The Biology of Art from 1963 and The Artistic Ape: Three Million Years of Art from 2013 as well as, among others, The Naked Ape or The Human Zoo, we should look for the earliest sources of art in events of special importance for the community such as festivals and rituals, like, for example, a successful hunt, birth, rituals of initiation (e.g. entry into adulthood), marriage act, war expeditions, death (burial), as well as remembrance of the dead and fear of superstitions. Morris refers here to Ellen Dissanayake's concept (1995, 2000; Morris, 2013), although it can be said with a high degree of probability that he had some influence on its development during their scientific cooperation in the late 1960s. According to this concept, the main reason for the emergence of art in the evolutionary history of humankind was the need to emphasize and highlight special and exceptional moments and events, but also make everyday events more unique (making special, artification). Recalling the experiment with the chimp Congo, Morris also claims that some higher primates, if properly taught, can create images that fit into the criteria of aesthetic perfection of human art.

\section{Congo Called Artist}

As the British anthropologist notes, at the age of 3 Congo had plenty of physical energy, was very curious and impulsive. A striking feature of his behavior was that when he received a pencil and began to draw, his excessive energy levels dropped. Morris, who studied many aspects of Congo's behavior, saw that it had its favorite shape: a beam of scattered, radial lines spreading out from the bottom of the page in every direction.

One of the surprising aspects of the drawing sessions with Congo was the intensity with which he worked. He did not receive rewards in the form of food, and creating drawings in itself was a reward for him. He was not interested in analyzing finished works but the act of creation fascinated him. He also knew at what point drawing should end. When he was being persuaded to continue, he refused, but when he received a new piece of paper, he immediately began to enjoy the opportunity to take on a new challenge. During several sessions in which, for some sudden reason, one needed to interrupt his work or interfere with an unfinished drawing, he reacted with screams and even with temper trantrums. As Morris writes, "It seemed extraordinary that a chimpanzee should be so upset when attempts were made to stop an activity as specialized as picture making. . . Why on earth should it have such a powerful appeal for an animal that shows no inclination to perform any-thing like it in the wild?" (Morris, 2013, p. 28). 
The drawings made by Congo belonged to three categories: drawings on an empty piece of paper, drawings on pieces of paper with geometric shapes and paintings on colorful cards. From the first moment he got the brush, it was obvious that Congo considered painting as more exciting than drawing with a pencil. Congo's drawing activity went through a total of three stages. In the first one the chimpanzee became acquainted with a new painting medium, in the second he gradually took control over brushes and was able to paint a thickened shape resembling a beam. In the third stage, which was broadcast live on the television, despite the distraction caused by the presence of the television crew, the chimp was very excited about the act of painting and creating images. Approximately during the $14^{\text {th }}$ painting session Congo showed that he had fully mastered the new medium and painted in a completely sure way. When observing him, it became clear that his every sign and line was placed exactly where he wanted it. The initially simple shape of the beam became more and more complex. Each line was carefully placed in relation to the others and the whole composition was designed to fit in a place intended for it. When on the next day Congo once again performed live on television, he was so confident that he painted a large and complicated beam shape (Morris, 2013).

Approximately around the $22^{\text {nd }}$ session that took place on September 2, 1957, Congo reached the peak of painting competence and control. No random sign appeared then on his paintings. Every line painted on the paper was put exactly where he wanted it to be and he used the available space with the dash of a professional human artist. He played with his beam pattern, tilting it one way, making a dotted version of it or splitting it into two parts. (Out of the ten paintings created that day, all were sold to private collections in Europe and North America, for example, Pablo Picasso and Joan Miro bought one painting each.)

In the following weeks, Congo continued to create abstract works of quality which had not yet been observed in any animal. Each time, he also explored new variations. He created a tilted beam, an additional beam, a beam with a curved base and 3 separate beams marked in the central part with a yellow, black and blue point respectively. He particularly liked the typically human aesthetic game, namely thematic variations. "Those who watched him during this stage simply sat in amazement, unable to believe what they were seeing." This peak period of Congo's artistic creation lasted until the end of 1957. Up to this point, he had created over 30 high-quality works. In 1958 he entered the third stage in which, despite the persisting boldness and self-confidence, his level of interest in the activity of painting began to decrease. Many of his works of this period were created quickly and intensively, but with less attention to detail. Energetic vertical shapes and spontaneous loops slowly replaced the developed beam patterns.

\section{Animal-Made Art - Capitulation of the Aesthetic Theory?}

Morris claims that the experiment with the chimpanzee Congo proves that not only humans but also other primates, if properly taught, can create images that demonstrate te ability to adhere to several basic aesthetic principles: feeling satisfaction with the very act 
of painting (the chimpanzee did not receive any reward); compositional control of the painting (Congo was able to limit his painting to a designated outlined space and maintain the compositional balance of the overall layout of the lines and pattern variations); calligraphic variation (on a completely basic level, with a much lower intensity than in young children); thematic variations and optimal heterogeneity of the image (neither too few nor too many shapes, lines, etc.) Although compositions drawn by chimpanzees are in fact better when it comes to the sense of rhythm and balance than compositions drawn by 2year-old children (Kellog, 1955), unlike them, the chimpanzee never reaches the imaging stage which the child enters at the age of 3-3.5, when he or she starts to represent his or her mom, dad, cat and house.

So, can Congo's paintings, as Morris wants it, be considered art? The greatest advantage of the analyses of the author of The Artistic Ape (apart from his thorough knowledge about primates), namely the excellent expertise in artistic theory and practice (the author is a recognized expressionist painter), also constitutes its weakness. The author is aware of the importance of contemporary painting practices and deliberately applies the definition of art taken from impressionism when referring to art in general. This makes it much easier to classify Congo's exceptional achievements as artistic (he calls Congo's style abstract lyrical impressionism). However, if we look at the nature of these achievements a little closer, it will turn out that they do not go beyond the aforementioned theoretical horizon.

\section{"A lot has happened in the meantime, both to their family and to ours"}

In his essay published in one of the Edge.org volumes Denis Dutton writes:

Consider Wittgenstein's gnomic, seemingly profound claim, 'If a lion could speak, we could not understand him.' Oh yeah? That's a deeply mischievous idea, and Wittgenstein would have profited from getting to know an animal ethologist or two. If a lion could speak, the ethologists would be pretty clear about that he'd be talking about: annoying other lions, and members of the opposite lion sex, tasty zebras, and so on. People who live with animals can understand them, sometimes rather remarkably. (Dutton, 2011, p. 55)

Dutton, the author of the monumental The Art Instinct, treats the issue of animal-made art rather marginally, devoting it a total of one paragraph in the Introduction and a small but significant piece in Art and Human Reality where he states that chimpanzees have fun scribbling or plotting vertical shapes. Still, the pleasure consists in simply filling the white background with a solid color and does not differ much from the pleasure of creating contrasts, which most of us have while smearing with our fingers or during first painting attempts at school (Dutton, 2011, pp. 55-56). However, it cannot be said with certainty that he includes in the marginal cases, together with the works of Duchamp, Schonberg's compositions or the final match of the soccer World Cup. While he successfully applies the criteria of his cluster concept of art to Dadaist and conceptual art or atonal music and the spectacle of a soccer final, the status of chimpanzee painting is settled quite clearly: "To call this art or proto-art underestimates and misunderstands what human art is" (Dutton, 2011, p. 56). 
According to Dutton, people who claim that there is such a thing as intentional artistic creations of chimpanzees are usually not aware of other aspects of primate behavior. First of all, the typical vertical shape that usually appears in such images is not really reproductive because the chimpanzee is unable to present it upside down. Second, if the caretaker does not take a piece of paper away from it in advance, the result of playing with the brush will inevitably be a dark brown spot, as the chimpanzee has no idea when to stop. It is also difficult to discern any purpose, sense of an action plan or an end point to which the work is heading. It only appears to have these qualities because the trainer took it from the chimpanzee in advance before it became a shapeless stain. Finally, and most meaningfully for Dutton, when chimpanzees finish painting or when a piece of paper is taken from them, they never come back to look at their work. Chimpanzees like to stain white paper with colored spots but it does not make them creators of art works, Dutton seems to say. "There is no cultural tradition within which chimps are working. There's no criticism-art talk or evaluation of any kind - with the chimps. There's no style in the sense that it's a learned way of doing it, though there are uniformities in the output for muscular reasons". And further: "It is seems to me that anyone who says, 'Yes, chimpanzees have art,' is making a mistake" (Dutton, 2011, p. 56).

This position of the author of The Art Instinct should not be surprising because it is a fairly obvious consequence of the theoretical perspective adopted by him (intentionalism, essentialism and aesthetic perceptualism) for at least two reasons. First, as noted by Joseph Carroll, Dutton defines the proper subject of his considerations - which fundamentally distinguishes him from Dissanayake (1988) - primarily as high art of developed civilizations (Carroll, 2010) described as the "white, cold peaks of art" by Clive Bell (Bell, 1958), and the "undisputed, paradigm cases" by Dutton himself:

Instead of asking how is it that Duchamp's readymades are works of art, I say, let's ask what is it that makes the Pastoral Symphony a work of art. Why is A Midsummer Night's Dream a work of art? Why is Pride and Prejudice a work of art? Let's look first at the undisputed paradigm cases and find out what they all have in common.... Better to understand them, and then analyze modernist experimentation and provocations, such as Duchamp's brilliant work (Dutton, 2011, p. 52).

Second, being an art anthropologist and a Darwinian naturalist, Dutton faultlessly recognizes the falseness of pseudo-scientific jargon, regardless of whether it originates from the postmodern anything goes, from unauthorized excursions of some comparative ethologists in search of linkages between the behavior of people and other animals (especially primates) or from a simple misunderstanding of the place art occupies in human reality:

The gulf between human and chimpanzee "art" should be no surprise: our ancestors branched off from theirs six millions years ago. The ensemble of adaptations that became the human art instinct go back in our prehistory only a hundred thousand years or so, a tinyone-sixtieth fraction of the time span back to our ancestral split with chimps. A lot has happened in the meantime, both to their family and to ours. (Dutton, 2009, p. 8) 


\section{"Another uniqueness claim bites the dust!"}

Dutton's argument, however, has a double-edged character which it paradoxically owes to the modern discoveries of evolutionists. The fact that we find more and more points of contact between the behavior of homo sapiens and other animals supports the position that some of the animals could be regarded creators of art (Davies, 2012, p. 30). Examples are provided particularly by primatology and comparative ethology. Numerous observations of animals engaging in activities - such as the production and use of tools - that until recently were regarded as uniquely (and characteristically) human point to an interspecific affinity rather than a complete break between the species. The same applies to certain mental predispositions such as emotionality or self-awareness which occur both in humans and other animals (although with varying intensity), so that in the light of discoveries of modern evolutionary sciences it is assumed that the difference between the human and other primates is, in principle, not qualitative but quantitative, and the interspecific universality of many characteristics and behaviors is indisputable in the opinion of primatologists, comparative psychologists and animal ethologists.

It is no different with the flagship concept in evolutionary psychology, but also revolutionary narrative and literary studies - namely the "theory of mind", i.e. the cognitive ability of an individual to accept the second-person perspective, enabling him to recognize mental states of other persons, track the trajectory of their actions and predicting behavior, socially important cheating skills. As it turns out, this ability is almost certainly not unique to homo sapiens as chimpanzees and bonobos are also equipped with it. "Another uniqueness claim bites the dust!" announced triumphantly the primatologist Frans de Waal on his Facebook profile, referring to the experiment (the results were published in Science in the October 6, 2016 issue) in which chimpanzees passed the classic test of attributing false beliefs to others (a modified version of the so-called Sally-Ann test) (Caruso, 2016).

Until now, the basic problem in the study of advanced cognitive abilities of chimpanzees, including the possibility of subjecting them to the false belief test, was the lack of an appropriate method that would allow to determine the focus of a chimpanzee's attention at a given moment. Lacking the faculty of speech, the chimpanzee cannot communicate to the researcher in a conventional way-i.e. using words (as would be the case of 2, 3, 4-year olds taking the test) — where it believes Sally will look for candies previously hidden by Ann. Therefore, the experiment conducted by researchers form the Max Planck Institute for Evolutionary Anthropology in Leipzig and Kyoto University's Kumamoto Sanctuary was primarily about finding a way to make the results independent of verbal communication as a source of information about the intentions of the research subject. This was achieved through the use of the innovative eye-tracking method tracks the trajectory of a chimpanzee's sight while it is subjected to the experiment. The key to the success of the experiment (confirming the hypothesis that the chimpanzee possesses the "theory of mind") was to capture the moment when the animal focuses its eyes on the spot where the individual it observed had hid an object before it was moved to a different place without the chimpanzee's knowledge. If the chimpanzee did not have the "theory of mind," its attention, like 
the attention of a typical human three-year-old, would be focused on the point to which the object was moved (unknown to the individual observed by the chimpanzee, but known the chimpanzee). The fact that the chimpanzee clearly expects that the observed individual will follow in the direction suggested by his or her (the individual's) outdated and erroneous belief about the hiding place proves that it understands that the observed individual may have different beliefs from its own and that they may be false beliefs.

The experiment of the researchers from Leipzig not only shows in a unique way the need to "avoid excessive dependence on language skills necessary to understand narratives and questions in testing the theory of mind in children," but also "emphasizes the mental continuity between apes and humans." (de Wall, 2016, p. 40) We can thus, following Tecumseh Fitch, consider it "the last nail in the coffin of the long-standing idea that humans are the only species with the "theory of mind"" (Caruso, 2016).

Does fact that chimpanzees possess the "theory of mind" (mind reading, empathic accuracy or, as G. Currie describes it - ability to mentalize?-mentalising), a skill necessary to create simple tools and engage in advanced social or "political" practices (Szymborski, 2011), also mean that they have artistic skills? Are chimpanzees therefore capable of creating art? Modern ethology provides a lot of evidence which at least at first glance, give grounds to answer this question affirmatively.

If we refer to the definition proposed by Tatarkiewicz in A History of Six Ideas - according to which art produces beauty, represents or reproduces reality, creates forms, expresses, produces aesthetic experiences and causes shock (Tatarkiewicz, 1980, pp. 27-33) it will turn out that Congo's paintings do not fall into this category. First of all, its drawings do not reproduce reality and do not give shape to things, which still, however, allows them to categorized as non-figurative painting. Moreover, nothing stands in the way, especially taking into account a certain artistry of images created during the $22^{\text {nd }}$ session and the emotional involvement of the chimpanzee, to include them in the category of artification activities which might support the hypothesis of making special, claiming that the most original function of art was to leave marks and mark-making, not copying, imitating or symbolizing (Dissanayake, 1995, 2013). Of course, with this qualification of proto-artistic activities of the chimpanzee, it is important to underline that the animal undertakes the activity of "marking," unlike primary people, as a result of a clear incentive from the caretaker; the activity is not motivated by the inner need for invention or preceded by investment of time, energy and hard to reach materials (e.g. multi-day expeditions to acquire rare dyes by hunters-gatherers).

The issues of aesthetic survival and shock induction also seem difficult to verify empirically (both are related to the intentionality of chimpanzee painting trials that is key to our deliberations). They can be caused by each of the components of the emotional response to the situation in which, as a result of encouragement, the chimpanzee was put (mechanical hand movements, reactions to colors, fulfilling the caretaker's request, etc.). 
The cluster definition introduced by Dutton also does not explicitly decide in favor of recognizing the effects of chimpanzee creative work as art. ${ }^{1}$ Considering the absolutely exceptional case of Congo, ${ }^{2}$ in which signs of intentional action could be observed, it cannot certainly be said whether chimpanzees and other animals "enjoy art for itself, not demanding that it should protect them from the cold, or provide them with food and matrimonial attractiveness" (criterion of direct, impractical pleasure). In many cases the opposite is true: bowerbirds decorate their nests only in order to attract the attention of females (and this is a strictly functional motivation); although Morris compares Congo's achievements from the $22^{\text {nd }}$ session to the impressionist style in painting ('style' criterion), which also did not escape the attention of Miro and Picasso who bought the paintings, this qualification seems to be exaggerated. Certainly, these works do not represent (imitate) the experience related to the real world ('representation' criterion). They are also not clearly separated from everyday life, treated as a source of specific experiences ('special focus' criterion) or seem to have been created to use complex and diverse perceptual and intellectual abilities in their full extent ('intellectual challenge' criterion). Surely, the criterion of art tradition and institutions is doubtful and not applicable here either, even with the assumption of some form of animalistic theory of mind or the ability to mentalize that animal "artists" and "art recipients" meet in fanciful worlds of imagination ('imaginative experience' criterion).

On the other hand, it cannot be unambiguously denied that among female spiders or bowerbirds there exists some kind of criticism and assessment of the "artistic achievement" of the male, be it a steady rhythm of tapping or visual improvement of the nest ('criticism' criterion). It may even be possible that the criteria of this criticism and assessment have been genetically fixed, being transmitted from generation to generation. There is a high probability that, for example, in the world of bowerbirds, similarly to the human world, the artistic or decorative craft ('skill and virtuosity' criterion) is cared for, appreciated and admired; what is perhaps also evaluated, praised and admired in the "work" is its originality, creativity and ability to surprise the audience even if it is an audience consisting only of female-connoisseurs of the same species ('novelty and creativity' criterion), although the functional nature of the achievement (courtship) accompanying it seems to contradict it. It also does not explicitly negate the "artistic aspirations" of animals, i.e. the ability to express the individual artistic personality accompanying their work and inherent in artistic practices, regardless of whether it is fully achieved (criterion of expressive individuality);

\footnotetext{
${ }^{1}$ Dutton creates a list of twelve "recognition criteria" of art (present inter-culturally and supra-historically), which, in his opinion, will facilitate an understanding of what art is as a universal human phenomenon in its diversity and indeterminacy. These criteria are (Dutton, 2009, pp. 51-59): (1) direct (impractical) pleasure, (2) skill and virtuosity, (3) style, (4) novelty and creativity, (5) criticism, (6) representation, imitation, (7) special focus, (8) expressive individuality, (9) emotional saturation, (10) intellectual challenge, (11) art traditions and institutions, and (12) imaginative experience.

${ }^{2}$ In fact, the claim that chimpanzees create art because Congo did it is equivalent to the claim that they know sign language because one of them, the chimpanzee named Washoe, learned and used around 250 signs of American Sign Language.
} 
nor does it negate that every artwork experience is intertwined with emotions of varying degrees of saturation ('emotional saturation' criterion).

Why then, according to popular opinion (and rather obvious intuition), a typical chimpanzee does not create art? There are at least several important arguments for such a position. First of all, the chimpanzee seems to disturb and destroy the white space on paper rather than fill it for aesthetic reasons (which is pointed out by, for example, Lenain, 1999). Second, the chimpanzee usually shows a lack of interest in the finished image after painting. Third, if the caretaker does not take the paper away at the right moment the image becomes a dark stain. The exception here is Congo who, during several sessions of the "mature period" showed optimal image heterogeneity, knowing exactly when to stop painting. Fourth, in any other case the pleasure resulting from the activity of painting seems to be derived from rhythmic movements, not from aesthetic causes (as pointed out by Davies, 2012).

What arguments are then in favor of attributing meaning to chimpanzee art? The answer cannot be given without a certain amount of cynicism. If the world of art recognizes that it is worth dealing with, then according to the institutional definition of art, nothing stands in the way of including chimpanzees, elephants, bowerbird nests and spider stepping in the concept. Chimpanzee painting is also similar to art as a natural category, a universal property of human mental capacity, one of the recognition criteria of its perfection, namely rarity of occurrence (Dutton, 2004). While human artists can be counted in millions (and using the broadened definition of art perhaps even billions), there are at most a few thousand chimpanzee artists. Just as we value rare materials, rare artifacts seem valuable to us. The least important is the charitable function of chimpanzee art (or animal-made art in general, just to mention images painted by elephants in the famous experiment by Komar and Melamid, conducted as part of the campaign to save elephants called the Asian Elephant Art and Conservation Project), usually emerging in institutions that operate on the basis of voluntary donations and take care of animals.

\section{References}

Bell, C. (1956). The aesthetic hypothesis. In Art. New York, NY: Art, Capricorn Books.

Carroll, J. (2010). The art instinct in its historical moment: A meta-review. The Evolutionary Review: Art, Science, Culture, 1 .

Caruso, C. (2016, October 6). Chimps may be capable of comprehending the minds of others, Scientific American. Retireved from https://www.scientificamerican.com/article/chimps-may-be-capableof-comprehending-the-minds-of-others/

Darwin, C. (1871). The descent of man, and selection in relation to sex. London, UK: John Murray.

Davies, S. (2012). The artful species: Aesthetics, art, and evolution. Oxford, UK: Oxford University Press. 
de Waal, F. (2001). The ape and the sushi master, New York, NY: Basic Books.

de Wall, F. (2016). Apes know what others believe, Science, 354(6308), 39-40.

Dissanayake, E. (1988). What is art for? Seattle, WA: University of Washington Press.

Dissanayake, E. (1995). Homo aestheticus: Where art comes from and why. Seattle, WA: University of Washington Press.

Dissanayake, E. (2000). Art and intimacy: How the arts began. Seattle, WA: University of Washington Press.

Dissanayake, E. (2000). Born to artify: The universal origin of picturing. In K. Sachs-Hombach \& J. R. J. Schirra (Eds.), Origins of pictures: Anthropological discourses in picture science (200-219). Köln, Germany: Halem Verlag.

Dutton, D. (2004). Let's Naturalize Aesthetics. Retrieved from http://aesthetics-online.org/?page=DuttonNaturalize

Dutton, D. (2009). The art instinct: Beauty, pleasure and human evolution. New York, NY: Bloomsbery Press.

Dutton, D. (2011). Art and human nature. In J. Brockman (Ed.), Culture: Leading scientist explore civilizations, art, networks, reputation and the on-line revolution (pp. 43-56). New York, NY: HarperCollins Publishers.

Kellogg, N. (1955). What children scribble and why, Palo Alto, CA: N-P Publications.

Lenain, T. (1999). Animal aesthetics and human art. In J. B. Bedaux \& B. Cooke (Eds.), Sociobiology and the arts (pp. 239-251). Amsterdam, the Netherlands: Rodopi.

Morris, D. (1963). The biology of art, London, UK: Methuen.

Morris, D. (2013). Artistic ape: Three million years of art. London, UK: Red Lemon Press.

Szymborski, K. (2011). Polityczne zwierzę, Warszawa: Biblioteka Polityki.

Tatarkiewicz, W. (1980). A history of six ideas: An essay in aesthetics, Warsaw: Springer. 